\title{
Study on pipeline photogrammetry in pipeline gallery modules in the SASOL project
}

\author{
Zhibo Wan ${ }^{1,2}$, Shengwen $\mathrm{Yu}^{1}$, Shangguo Liu ${ }^{1}$, Ganggang Gao ${ }^{3}$, Youqiang Dong ${ }^{3}$ \\ ${ }^{1}$ School of Surveying and Mapping Science and Engineering, Shandong University of Science and Technology, Qingdao \\ 266590, China \\ ${ }^{2}$ School of Data Science and Software Engineering, Qingdao University, Qingdao \\ 266071, China \\ ${ }^{3}$ Qingdao Haily Measuring Technology Co., Ltd., Qingdao \\ 266061, China
}

Keywords: SASOL; pipeline quantitation; industrial photogrammetry; Electronic Distance Measuring Device (EDM)

\begin{abstract}
This study addresses the problem of pipeline opening measurement in SASOL pipeline gallery modules by proposing the use of an industrial photogrammetry method. The position coordinates of the pipeline opening in the local coordinate system were first obtained and then converted to the overall modular coordinate system using the common point. Experiments showed that the coordinates obtained from industrial photogrammetry had an internal accord accuracy better than $0.2 \mathrm{~mm}$, which satisfies the accuracy requirements for pipeline measurement. Compared with the electronic distance measuring device (EDM) method, the industrial photogrammetry method reduced the time taken for one measurement from $21.6 \mathrm{~h}$ to $1.5 \mathrm{~h}$, which substantially increases the measurement efficiency of the method, thereby increasing its application value.
\end{abstract}

\section{Introduction}

The South African SASOL Company is a world-famous petrochemical company with a large-scale gas-to-liquid (GTL) factory in Louisiana, USA. This factory is also one of the largest currently being built in the US ${ }^{[1]}$. Two-thirds of the pipeline gallery modules in the SASOL project are being built in China by the FTI (a consortium of Technip and Flou) engineering management company.

This project has relatively higher requirements for dimension measurements. For example, the dimension measurements of the steel module framework structure, including measurements of single module dimensions and module docking measurements, have a measurement accuracy requirement of $\pm 3 \mathrm{~mm}$, as do the pipeline measurements, including the coordinate measurements of the mouth of the pipelines in a single module and dislocation measurement of the openings during module docking.

\section{Current status of pipeline measurement}

The pipelines are distributed in various platforms on 1-4 layers on modules, as shown in fig. 1. Currently, pipeline measurement is carried out using electronic distance measuring devices (EDMs). However, this is limited by the operating environment of the site; for instance, if the EDM is setup on the ground surface, it is unable to see the pipeline openings (fig. 2).

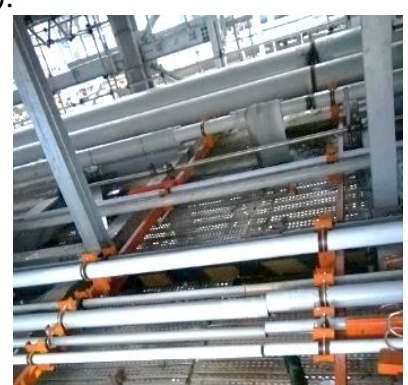

Fig. 1. Pipeline distribution map

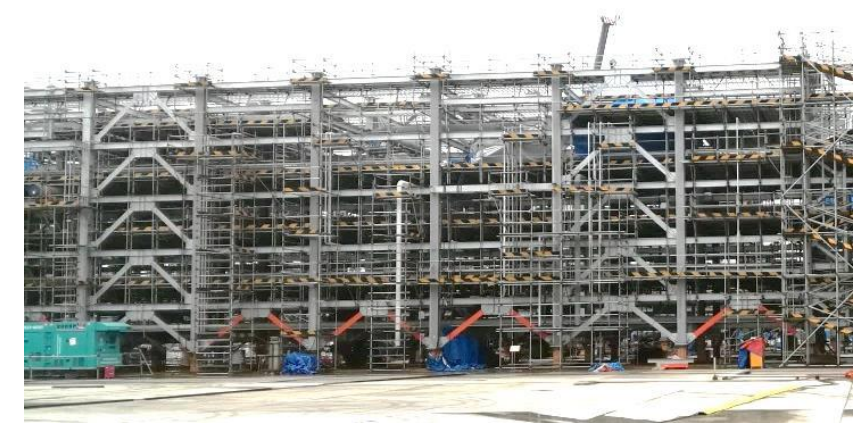

Fig. 2. Site map of pipeline gallery module

Therefore, when measuring pipeline openings, the modules must be separated and measurements must be carried out from the sides. The measurement process is as follows:

(1) After cutting the various pipeline layers along the crosssection, a crane is used to separate the modules.

(2) An EDM is setup on the ground frame to measure the control points of various modules in order to restore the local coordinate system. 
(3) In the local coordinate system, the EDM is used with a reflector to measure the point coordinates of various pipeline openings. Furthermore, fitting is carried out to obtain the center coordinates of the cross-section of the pipeline opening and to guide adjustment.

(4) The crane is used to rejoin and dock the various modules.

As the modules are bulky and the use of cranes is required to separate and rejoin them, the measurement process is timeconsuming, laborious, and expensive. Therefore, this study proposes the use of an industrial photogrammetry system with the EDM measurement method to solve problems in online measurements of pipelines.

\section{Principles of industrial photogrammetry}

The industrial photogrammetry system is an industrial measurement system based on the spatial angle intersection principle that is used to obtain the three-dimensional coordinates of measured points. This system uses a highresolution digital camera as a sensor, which gives a high quality "quasi-binary value" image when used with reflected marks. High accuracy three-dimensional coordinates are automatically obtained after image processing, image automatic orientation, automatic matching of image points, and self-calibrating bundle block adjustment. Currently, typical measurement accuracies of industrial photogrammetry systems can reach $\pm 0.1 \mathrm{~mm} / 10 \mathrm{~m}^{[2,3]}$.

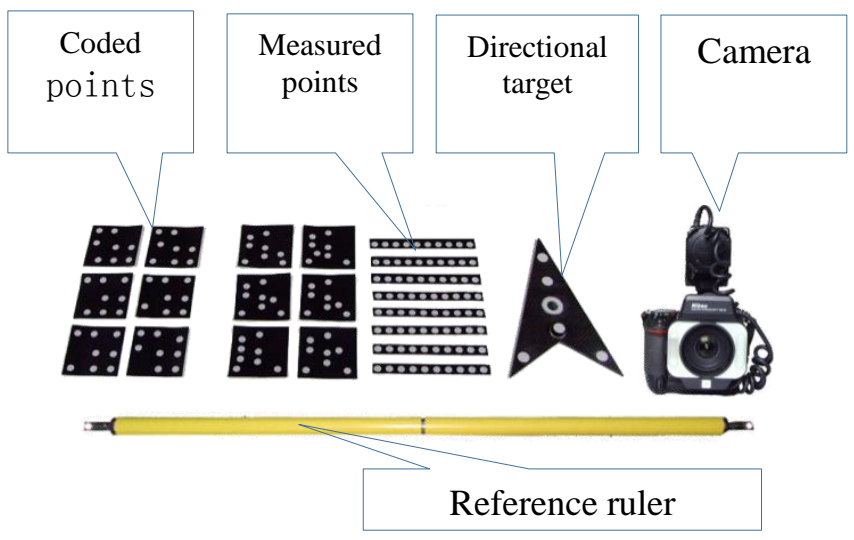

Fig. 3. Hardware components of an industrial photogrammetry system

An industrial photogrammetry system has characteristics such as high accuracy, high efficiency, automation, flexibility, and convenience. It is suitable for use in the high accuracy measurements of shapes and dimensions of various industrial products, and has become widely used in industrial manufacturing in recent years. As this method uses handheld cameras to capture images, it does not require a stable observation platform. Therefore, it is particularly suitable for measurements in environments with narrow spaces and multiple obstacles ${ }^{[4]}$.

\section{Pipeline industrial photogrammetry method}

\subsection{Layout of marker points and accessories}

To obtain the position of the center location of the pipeline section, markers were pasted evenly throughout the section for fitting of the section center, as shown in fig. 4 .

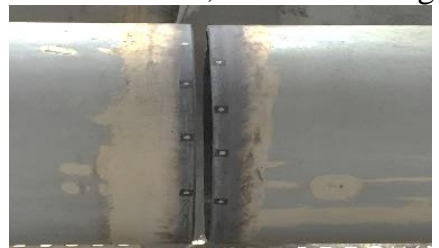

Fig. 4. Placement of markers

Coded points were evenly placed on the pipeline and the beam used for fixing the pipeline to orient the image, as shown in fig. 5. The reference ruler and the directional target were both fixed on the center pipeline.

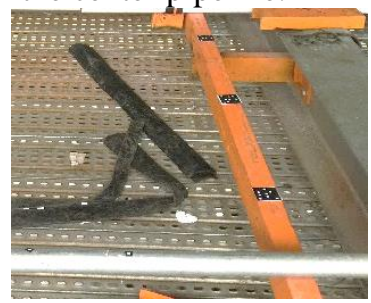

Fig. 5. Placement of coded markers

The overall placement results of the markers and accessories are shown in fig. 6.

\subsection{Setup of camera station}

Photographs were taken at a distance of $1 \mathrm{~m}$ from both sides of the pipeline opening, with the interval between the two neighbouring shooting positions controlled at around $1 \mathrm{~m}$. As shown in fig. 7 , a photograph each was taken from the left frontal position, the direct frontal position, and the right frontal position. For every measurement, approximately 120 photographs were taken.

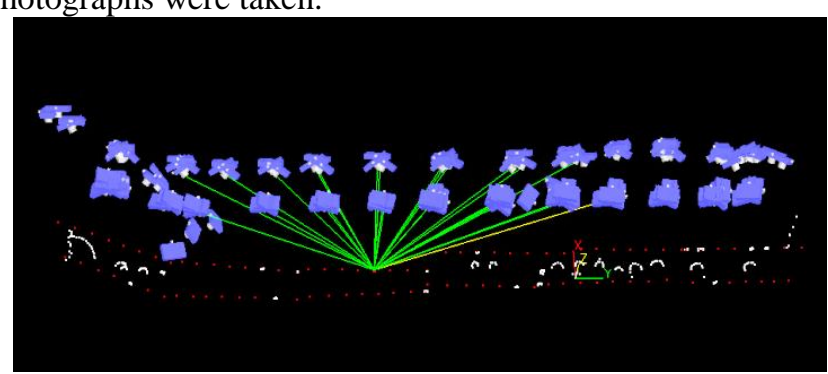

Fig. 7. Schematic of the distribution of the camera station

\subsection{Conversion of coordinate system}

The measurement coordinate system used by the industrial photogrammetry system is a local coordinate system. In order to convert the coordinates of the measured points into the overall modular coordinate system, some coordinate points from the EDM were used as common points ${ }^{[5]}$. The method is as follows: 
(1) Four reflector tools (rotatable) were fixed at both sides of the cross section on every layer of the module for EDM use. Each tool was pasted with a photogrammetry marker so that the center of each marker coincided with the center of the reflector, as shown in fig. 8 .

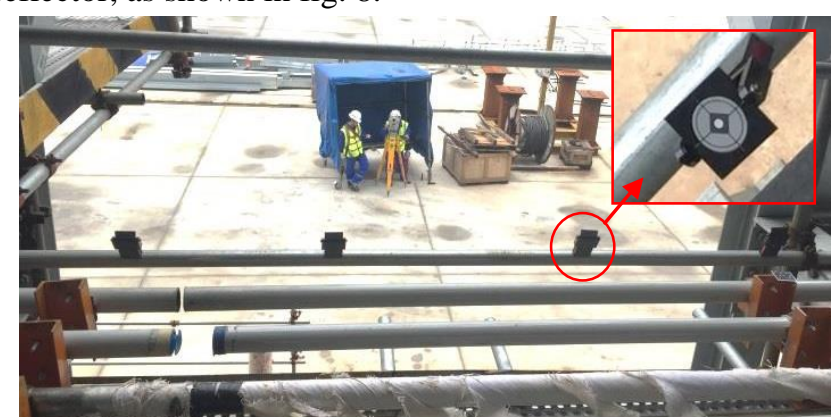

Fig. 8. Interchangeable tooling for the electronic distance measuring device (EDM) and photogrammetry

(2) The tooling was rotated toward the ground surface and the EDM was used to measure the 3-D coordinates of the tooling point in the overall coordinate system of the module.

(3) The tooling was rotated diagonally upward, and photogrammetry was used to measure the coordinates of the pipeline ends and the 3-D coordinates of the tooling point in the photogrammetry coordinate system simultaneously.

(4) Through conversion using the common point, the pipeline end coordinates were converted into the overall coordinate system of the module.

\section{Experiment and conclusions}

Pipeline measurements were carried out at the docking sites in the second layer of the SASOL pipeline galleries $40 \mathrm{C}$ and 40E. A total of four groups and eight pipeline opening locations were measured, and 153 measuring points and 40 coding points were set up. The Nikon D810 camera was used to take a total of 116 photographs.

fig. 1 shows the internal accord accuracy (accuracy estimate) of the coordinates after photogrammetry data processing.

Table I Accuracy statistics of photogrammetry

\begin{tabular}{|c|r|r|r|r|}
\hline Statistics & $\mathrm{X}$ & $\mathrm{Y}$ & $\mathrm{Z}$ & Total \\
\hline RMS $(\mathrm{mm})$ & 0.017 & 0.019 & 0.032 & 0.041 \\
\hline Maximal value $(\mathrm{mm})$ & 0.081 & 0.059 & 0.122 & 0.150 \\
\hline $\begin{array}{c}\text { Residual of image } \\
\text { point coordinates }(\mu \mathrm{m})\end{array}$ & \multicolumn{5}{|c|}{0.62} \\
\hline
\end{tabular}

The measured coordinates were inputted into the DACS software, which can immediately and automatically generate a report on the design deviation and docking deviation of the pipeline opening, as shown in fig. 9.

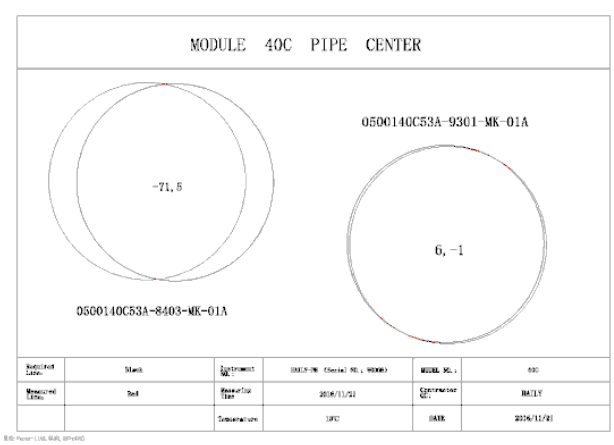

Fig. 9 Pipeline opening measurement report

Table I compares the time taken to complete specific activities when the photogrammetry and conventional EDM measurement are utilized.

Table II Time taken to complete specific activities when each of the measurement methods is applied (unit: h)

\begin{tabular}{|c|c|c|c|}
\hline \multicolumn{2}{|c|}{ Photogrammetry } & \multicolumn{2}{c|}{$\begin{array}{c}\text { Electronic Distance } \\
\text { Measuring Device (EDM) }\end{array}$} \\
\hline Work content & $\begin{array}{c}\text { Time } \\
\text { taken }\end{array}$ & Work content & $\begin{array}{c}\text { Time } \\
\text { taken }\end{array}$ \\
\hline Marker layout & 0.5 & Module separation & 10 \\
\hline Image capturing & 0.2 & Setting up EDM & 0.5 \\
\hline Data processing & 0.2 & EDM measurement & 1.0 \\
\hline $\begin{array}{c}\text { Measurement of } \\
\text { control points }\end{array}$ & 0.5 & Report generation & 0.1 \\
\hline Report generation & 0.1 & Module joining & 10 \\
\hline Total & 1.5 & Total & 21.6 \\
\hline
\end{tabular}

From the above experimental results, we can draw the following conclusions:

(1) The internal accord accuracy of the industrial photogrammetry measurement is better than $0.2 \mathrm{~mm}$, which can fulfill the accuracy requirements of pipeline measurements from pipeline gallery modules.

(2) Compared with the conventional EDM measurement method, the industrial photogrammetry method does not require module separation, which substantially decreases measurement costs and increases measurement efficiency at the same time, and has good application value.

\section{Acknowledgment}

This work is supported by the higher doctoral research fund (20093718110002), all support is gratefully acknowledged.

\section{References}

[1] China National Petroleum Corporation. Sasol invests in ethane cracking and GTL projects in the United States. http://www.pujyt.com/article/201412/20141230619.html. [2] Qi-qiang F, Guang-yun L and Zong-chun L. Digital industrial photogrammetry technology and application, Surveying and mapping press. Beijing, (2013).

[3] Koelman H J. "Application of a photogrammetry-based system to measure and re-engineer ship hulls and ship parts: An industrial practices-based report", Computer-Aided Design, 42, pp. 731-743, (2010). 
[4] Qi-qiang F, Bai-xing F and Qiang L., "Research on Satellite Deformation Measurement Technology in Satellite Coordinate System", Bulletin of Surveying and Mapping, S1, pp. 97-98, (2014).
[5] Feng-bin W, Xiao-feng L and Sen Z., "The Research of Joint Alignment Test Method Based on Switching Principle of Common Point", Space Electronic Technology, 13, pp. 9194, (2008).

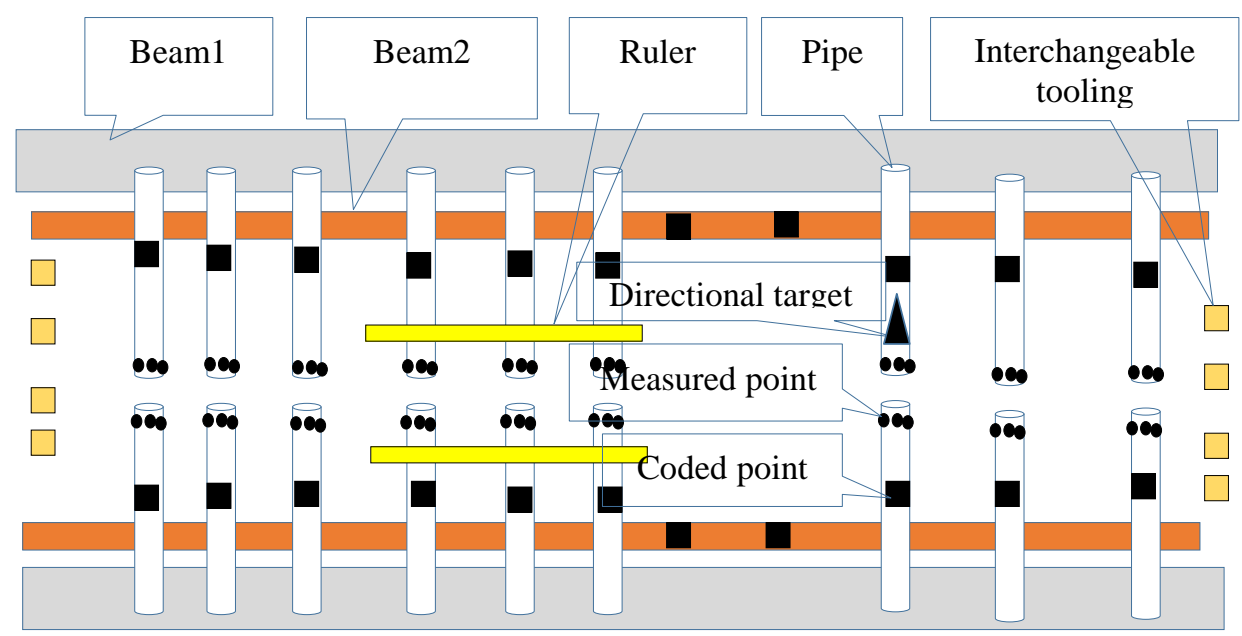

Fig. 6. Schematic of marker and accessories placement 